\title{
Using Origin Software to Study Silicon Effect on Yunnan Building Phosphogypsum
}

\author{
Yihui Yao ${ }^{1, a}$ Zhiman Zhao ${ }^{1, b}$ Sichen Quan ${ }^{2, \mathrm{c}}$ Wenjia Zhang ${ }^{1, \mathrm{~d}}$ Feng Gao $^{1, \mathrm{e}}$ \\ $1,{ }^{*}$ Kunming University of Science and Technology, Kunming, Yunnan \\ ${ }^{2}$ Yunnan Kunming Steel Structure Limited Company, Anning ,Yunnan

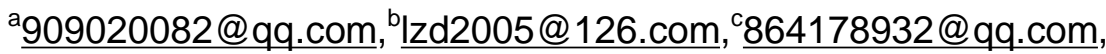 \\ d418843486@qq.com, ${ }^{\mathrm{e}}$ 654293136@qq.com
}

Keywords: silicone defoamer, compressive strength, setting time, data fitting

Abstract:This paper had explored the effects of different contents of silicone defoamer for time of coagulation and compressive strength of building phosphogypsum. At last, this paper had used origin software to deal with the data of test and verified fitting equation.

Phosphogypsum was a by-product of wet phosphoric acid industrial production and the every 1t phosphate will produce $4.5 \mathrm{t}$ phosphogypsum in average, its main component was $\mathrm{CaSO}_{4} \cdot 2 \mathrm{H}_{2} \mathrm{O}$. Industrial by-product phosphogypsum have many uses, such as cement retarder, adsorption-based sludge, preparation construction materials and so on. With the developing of industrialization, the annual production of phosphogypsum by-product was also increasing, how to consume a lot of phosphogypsum was a major problem. Building materials was a great consumption products, even after pretreatment removal the most impurities of phosphogypsum, but the test blocks of building phosphogypsum contains a large number of bubbles, which can reduce its strength and influence on the mechanical performance of building phosphogypsum. So, this paper based on this phenomenon, studying silicone defoamer which its effects on the performance of building phosphogypsum in Yunnan province, aim at solving the problem of its porosity. Because silicone defoamers used for the textile industry, paper industry and so on, in building materials especially building phosphogypsum materials has little research in this area, Therefore, this study explore a new way to chose a better defoamer of building phosphogypsum.

\section{Experiment materials}

Phosphogypsum: Producted by Yuntianhua Co, which was pale yellow powder and has slight smell, its chemical component was shown in Table 1.

Table 1 Phosphogypsum component.

\begin{tabular}{ccccccccccccc}
\hline component & $\mathrm{SiO}_{2}$ & $\mathrm{Al}_{2} \mathrm{O}_{3}$ & $\mathrm{TFe}_{2} \mathrm{O}_{3}$ & $\mathrm{MnO}$ & $\mathrm{MgO}$ & $\mathrm{CaO}$ & $\mathrm{K}_{2} \mathrm{O}$ & $\mathrm{P}_{2} \mathrm{O}_{5}$ & $\mathrm{SO}_{3}$ & $\mathrm{Cl}^{-}$ & $\mathrm{F}^{-}$ & organics \\
\hline content/\% & 14.52 & 1.66 & 0.15 & 0.005 & 0.17 & 31.94 & 0.22 & 0.94 & 45.38 & 0.027 & 0.12 & 0.25 \\
\hline
\end{tabular}

Reagents: silicone defoamer(analysis of pure), citric acid(analysis of pure), solution of $\mathrm{Ca}(\mathrm{OH})_{2}$. Experiment equipments: Screening machine, libra, $\mathrm{PH}$ tester, oven, vibrating table, setting time tester.

\section{Experiment method}

\section{Preprocessing stage}

The phosphogypsum raw material of Yunnan were taken to through 100 mesh and by phosphogypsum: water $=1.5: 1$ the proportion of adding water, after stirred fully, then scrapped the black oily floater (which was mainly soluble organic impurities) on the surface of the water, this procedure was repeated 2 or 3 times until the surface of the water have no black oily floater after 
stirred phosphogypsum. After added of $0.35 \%$ citric habit modifier and until stirred evenly, then added $\mathrm{Ca}(\mathrm{OH})_{2}$ solution reagent to prepare a range of phosphogypsum raw material was $\mathrm{PH} \approx 7$. The deployment of a good phosphogypsum raw was poured into a iron laboratory dish, and placed it in an oven to bake three and a half hours under the conditions of $140^{\circ} \mathrm{C}$, and then removed it from the oven, used sieving machine through a 100-mesh .

\section{Paste stage}

First, the control group test was processed that adding standard consistency water in Yunnan building phosphogypsum materials which has been pretreated, then made paste stage after rapid stirred until it got gel state; for instrance, different contents of organic silicon were added to the building phosphogypsum materials under standard consistency water, and continue rapid stirred until it got gel state. At last, we were pouring it into vibrating table for vibrate uniform to finish the paste stage.

\section{Measure setting time and strength}

(1).According to 《Building gypsum mechanical property measurement $》$ measure its compressive and flexural strength.

(2) According to 《cement standard consistency water, setting time and soundness test method》 measure its setting time.

\section{Test results and analysis}

\section{Influence of different amount of organic silicon on the strength of Yunnan building phosphogypsum}

The compressive strength of the test block in the blank control group was $7.6 \mathrm{MPa}$, the initial setting time was $3.5 \mathrm{~min}$, and the final setting time was $5 \mathrm{~min}$. Under neutral condition, the experiment results of adding different content of organic silicon were shown in figure 1.

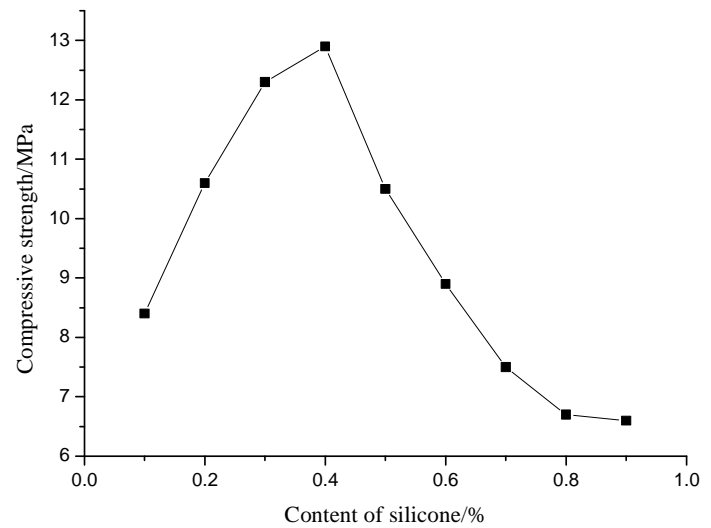

Fig1 Different contents of silicon to effect the strength of Yunnan building phosphogypsum

From the figure 1 , when the dosage was $0.4 \%$, Yunnan building phosphogypsum test block strength highest, the compressive strength was $12.9 \mathrm{MPa}$, and organosilicon reagents was added to Yunnan building phosphogypsum strength as a whole higher than that in the blank control group, this was because, silicone as a defoaming agent was added to the phosphogypsum that reduced the porosity of the block, thereby increased test block strength. Defoaming agent was mainly composed of silicone grease agent, waterproofing agent, thickening agent with proper amount of water emulsion made of machinery. Silicone fire foam agent and standard consistency water added to Yunnan Phosphorus building gypsum raw material, silicone molecules disorderly distribution in the liquid, inhibit formation of elastic film, to prevent the production of bubbles in the liquid of phosphorus building gypsum. In terms of the Yunnan Phosphorus building gypsum that had liquid bubble, silicone molecules spread on the surface of the bubble, the bubble surface and the formation of a layer of thin film, and further extrusion, infiltration in bubbles, so that the original bubble 
membrane rupture, produced in the process of internal and external pressure difference, the bubble rupture, this was the "bubble breaking" phenomenon. Silicone defoaming agent, greatly reduced the Yunnan Phosphorus building gypsum liquid in the bubble phenomenon, thereby reduced the porosity, improved inter block molecular adaptation, to enhance the Yunnan Phosphorus building gypsum block strength test.

Effect of different amount of organic silicon on the setting time of Yunnan building phosphogypsum

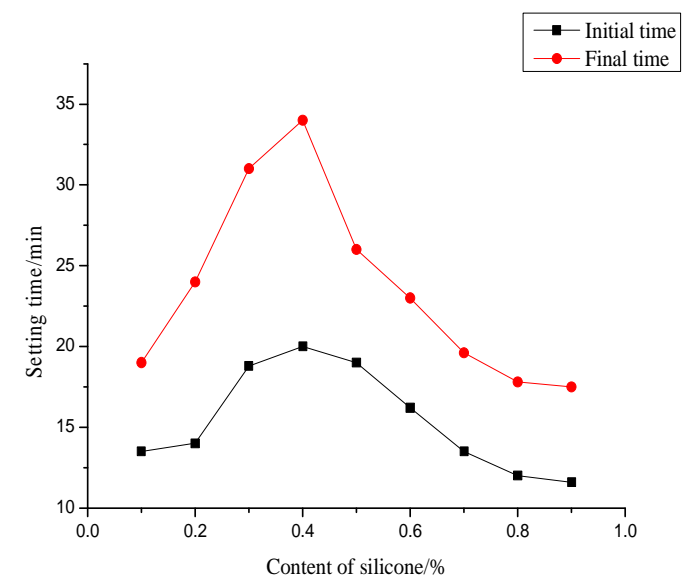

Fig2 Different contents of silicon to effect the setting time of building phosphogypsum

From Figure2, the lowest initial setting time was $12 \mathrm{~min}$, the lowest final setting time for $17.5 \mathrm{~min}$, the longest initial setting time was in silicone content $0.4 \%$, the initial setting time of $20 \mathrm{~min}$, eventually setting time was $34 \mathrm{~min}$, which than the control under the condition of the initial setting more $3.5 \mathrm{~min}$, eventually setting time was longer than $5 \mathrm{~min}$, organosilicon reagents had a slow coagulation and strength loss rate was lower were found through this. This was because on the one hand, silicone with high surface activity and had $\mathrm{Si}-\mathrm{O}-\mathrm{Si}$ ( $\mathrm{Si}-\mathrm{Si}, \mathrm{Si}-\mathrm{C}-\mathrm{Si}$ ) hydrophobic groups, it can better reduced the surface tension, phosphorus building gypsum hydration reaction rate decreased when a certain amount of silicone were added, so as to prolong the setting time. On the other hand, silicone with defoaming effect, in the pretreatment of phosphorus building gypsum added the right amount of silicone, the bubble rate decreased, the strength increased. Therefore, although the addition of organic silicon can prolong the time of the phosphorus building gypsum slurry, the strength loss rate was small.

\section{Origin data fit and analysis}

data fitting and analysis of compressive strength of Yunnan building phosphogypsum with organic silicon

The nonlinear relationship between the content of the added organic silicon and the compressive strength of the test block of Yunnan building phosphogypsum can be seen in Figure 1. The equation (1) was obtained by using the Origin software to fit the nonlinear Guass equation.

$$
\mathrm{y}_{1}=\frac{2.56}{0.33 \sqrt{\frac{\Pi}{2}}} e^{-2 \frac{(x-0.36)^{2}}{0.33^{2}}}+6.62
$$

And through the Origin software analysis of the validity of the fitting equation, its goodness of fit (Adj.R-Square) was 0.98792, the sum of squares of the residuals (Chi-Sqr Ruduced) was 0.06539 , which were showed that the fitting equation was higher. The fitting curve was shown in figure 3 . 


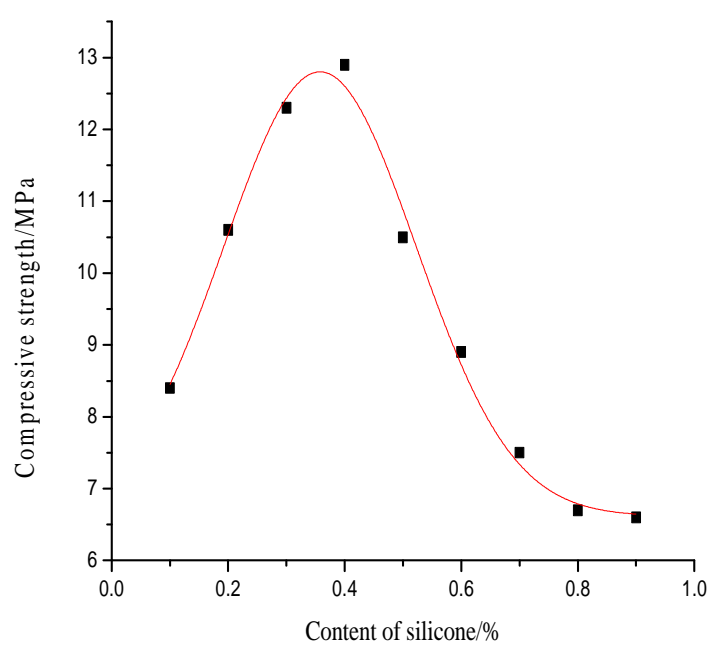

Fig3 The fitting curve of the compressive

And equation (2) can derived by equation (1).

$$
\mathrm{y}_{1}^{\prime}=\frac{2.56}{0.33 \sqrt{\frac{\Pi}{2}}} e^{-2 \frac{(x-0.36)^{2}}{0.33^{2}}} * \frac{-4}{0.33^{2}} *(x-0.36)
$$

\section{data fitting and analysis of initinal setting time of Yunnan building phosphogypsum with organic silicon}

The nonlinear relationship between the content of the added organic silicon and the initinal setting time of the test block of Yunnan building phosphogypsum can be seen in Figure1. The equation (3) was obtained by using the Origin software to fit the nonlinear Guass equation.

$$
\mathrm{y}_{2}=\frac{3.37}{0.31 \sqrt{\frac{\Pi}{2}}} e^{-2 \frac{(x-0.42)^{2}}{0.31^{2}}}+11.69
$$

And through the Origin software analysis of the validity of the fitting equation, goodness of fit (Adj.R-Square) was 0.96108, the sum of squares of the residuals (Chi-Sqr Ruduced) was 0.39647, which was showed that the fitting equation is higher. The fitting curves were shown in figure 4 .

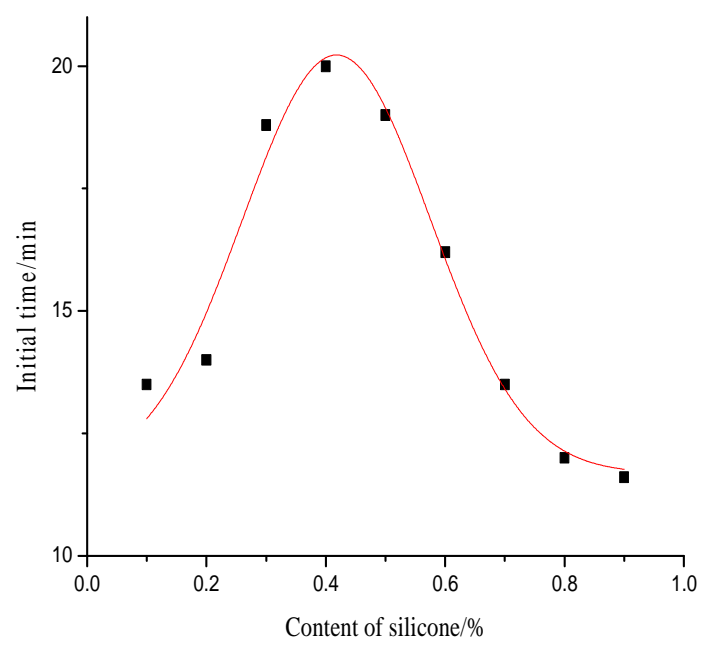

Fig4 The fitting curve of the initinal time

Through equation (3) the equation (4) was got as bellow.

$$
\mathrm{y}_{2}^{\prime}=\frac{3.37}{0.31 \sqrt{\frac{\Pi}{2}}} e^{-2 \frac{(x-0.42)^{2}}{0.31^{2}}} * \frac{-4}{0.31^{2}} *(x-0.42)
$$


By calculation, when $\mathrm{x}_{2}=0.42$ was obtained, $\mathrm{y}_{2}$ was zero and the maximum value of $20.39 \mathrm{~min}$ was obtained by equation (3). When the content of the organic silicon reagent was $0.42 \%$, the initial setting time of Yunnan phosphorus building gypsum was the longest, and its theoretical value was $20.39 \mathrm{mim}$.

The fitting and analysis of the final setting time data of Yunnan building phosphogypsum with organic silicon

Similarly, from figure 2 can be obtained the nonlinear relationship between the content of the added organic silicon and the final setting time of phosphorus building gypsum. Through nonlinear Guass fitting by Origin software, equation (5) was got.

$$
\mathrm{y}_{3}=\frac{4.93}{0.26 \sqrt{\frac{\Pi}{2}}} e^{-2 \frac{(x-0.38)^{2}}{0.26^{2}}}+18.09
$$

And through the Origin software analysis of the validity of the fitting equation, its goodness of fit (Adj.R-Square) was 0.94687, the sum of squares of the residuals (Chi-Sqr Ruduced) was 1.55245, which were showed that the fitting equation was higher. The fitting curve was shown in figure5.

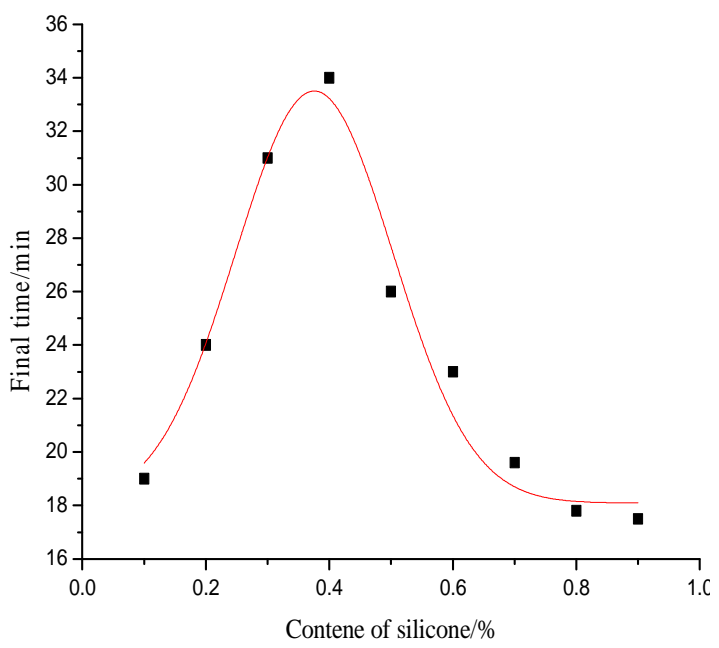

Fig 5 The1 ftting curve of the final time

The equation(6) can be calculated by equation (5).

$$
y_{3}^{\prime}=\frac{4.93}{0.26 \sqrt{\frac{\Pi}{2}}} e^{-2 \frac{(x-0.38)^{2}}{0.26^{2}}} * \frac{-4}{0.26^{2}} *(x-0.38)
$$

By calculation, it was obtained that when $\mathrm{x} 3=0.38$ the $\mathrm{y} 3=0$, yield the equation (5) obtains the maximum value of $33.26 \mathrm{~min}$. When organic silicon reagent was added to $0.38 \%$, the final setting time of Yunnan phosphorus building gypsum was the longest, and its theoretical value was 33.26min.

\section{Equation fitting validation}

By equation (1), (3), (5) and the derivative of equation (2), (4), (6), when the $\mathrm{y}_{1}, \mathrm{y}_{2}$ and $\mathrm{y}_{3}$, respectively to obtain maximum value, which was different from the corresponding $\mathrm{x}_{1}, \mathrm{x}_{2}, \mathrm{x}_{3}$, and the average of $\mathrm{x}_{1}, \mathrm{x}_{2}$ and $\mathrm{x}_{3}$ was 0.39 , calculated value $\mathrm{y}_{1}=12.83 \mathrm{MP}, \mathrm{y}_{2}=20.39 \mathrm{~min}, \mathrm{y}_{3}=33.26 \mathrm{~min}$. then in the laboratory silicone defoaming agent added to $0.39 \%$ in ten groups of test, it can be seen that the fitting equation (1) (2) (3) calculated and the actual test data error was very small, therefore, the fitting equation (1) (2) (3) can be used.

\section{Conclusion}

(1) Organosilicon defoaming agent widely used for paper making industry, textile industry and so 
on, but seldom applied in building industry, especially the phosphorus gypsum. Therefore, the study of its effect on properties of Yunnan building phosphogypsum is very far-reaching significance.

(2) At $\mathrm{pH}=7$, add in Yunnan Phosphorus building gypsum of organosilicon reagents showed the highest activity, that was, to play a role.

(3) An organosilicon defoaming agent with high surface activity. Its active surface with hydrophobic groups can reduce the hydration rate of Yunnan Phosphorus building gypsum paste, so as to prolong the setting time, so organic silicon anti foaming agent of Yunnan Phosphorus building gypsum has some retarding around.

(4) In adding standard consistency water, organosilicon reagents (optimum dosage of $0.39 \%$ ) is added to the Yunnan building phosphogypsum raw materials after the pretreatment, silicone molecular motion in the colloid, inhibit formation of the elastic membrane, to prevent air bubbles; also attached to have been produced around the bubbles, through inside and outside pressure to "broken bubble," the purpose, and ultimately makes Yunnan building phosphogypsum test block air rate is reduced, the strength is increased.

\section{Acknowledgements}

This work was financially supported by Natural Science Foundation of China(51264017), contract of scientific research of Steel Structure Company of Yunnan Kunming.

\section{References}

[1] Shumei Yin,Zhihao Wang.The study of efficient silicone defoamer[J].Silicone material,2010, 24(4):218-221

[2] Guoyun Zhang.Foams and defoamal technology in pulp and paper industrial producing processes[J].Heilongjiang Pulp \& Paper,2004,1:19-20

[3] Ranran Zhang,Gongzhao Liu.Research progress and application of organic silicon defoamer [J].Technology \& development of chemical industry,2015,44(8):20-24

[4] Tao Gong.Research progress of oganosilicon defoamer for textile[J].Chinese journal of colloid \& polymer,2010,28(2):94-96

[5] Yusuo Zhao.Organosilicon defoaming agent[J].Zhe jiang chemical industry,2007,38(3):12-15

[6] Chen Yang,Zhengyang Huang,Yang Zhang,Qi Xu etal.Comparative analysis on growth curve fitting of three types of goose[J].Chinese journal of animal science,2014,50(9)

[7] Mingzhu Sun, Yamin Jia,Hongli Wang, Qinggele Wu.Application research of software origin in experimental data processing[J].Research and exploration in laboratory,2015,34 (10) : 96-98

[8] Baoliang Liu,Guiping Cao.Application of origin software in data processing of distillation experiment[J].Higher education in chemical engineering,2015 (1) : 90- 91

[9] Min Li,Yi Sheng.Study on application of gaussian fitting algorithm to building model of spectral analysis[J].Spectroscopy and spectral analysis,2008,28(10)

[10] Virginia Raymond thiminur.New developments in alkyl silicones[J].Cosmetics \& toiletry, 1993,5-87

[11]Jianlong Huang.Presentation of organic silicione defoamer in brief[J].Synthetic lubricants, 2013(1):30-33 